\title{
Utility Assessment of a Map-Based Online Geo-Collaboration Tool
}

\author{
Christopher L. Sidlar
}

University of Toronto

\section{Claus Rinner}

Ryerson University

Sidlar, C. L., \& Rinner, C. (2009). Utility assessment of a map-based online geocollaboration tool. Journal of Environmental Management, 90(6), 2020-2026.

doi:10.1016/j.jenvman.2007.08.030

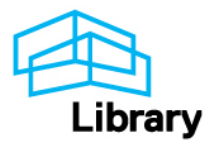




\title{
Utility assessment of a map-based online geo-collaboration tool
}

\author{
Christopher L. Sidlar ${ }^{\mathrm{a}}$, Claus Rinner ${ }^{\mathrm{b}, *}$ \\ ${ }^{a}$ Department of Geography and Program in Planning, University of Toronto, 100 St. George Street, Toronto, ON M5S 3G3, Canada \\ ${ }^{\mathrm{b}}$ Department of Geography, Ryerson University, 350 Victoria Street, Toronto, ON M5B 2K3, Canada
}

\section{A R T I C L E I N F O}

\section{Article history:}

Received 6 October 2006

Received in revised form 15 April 2007

Accepted 24 August 2007

Available online 9 June 2008

\section{Keywords:}

Argumentation Map

Geo-collaboration

Geographic information science

Planning support system

Public participation geographic information

system (PPGIS)

Software utility

\begin{abstract}
A B S T R A C T
Spatial group decision-making processes often include both informal and analytical components Discussions among stakeholders or planning experts are an example of an informal component. When participants discuss spatial planning projects they typically express concerns and comments by pointing to places on a map. The Argumentation Map model provides a conceptual basis for collaborative tools that enable explicit linkages of arguments to the places to which they refer. These tools allow for the input of explicitly geo-referenced arguments as well as the visual access to arguments through a map interface. In this paper, we will review previous utility studies in geo-collaboration and evaluate a case study of a Web-based Argumentation Map application. The case study was conducted in the summer of 2005 when student participants discussed planning issues on the University of Toronto St. George campus. During a one-week unmoderated discussion phase, 11 participants wrote 60 comments on issues such as safety, facilities, parking, and building aesthetics. By measuring the participants' use of geographic references, we draw conclusions on how well the software tool supported the potential of the underlying concept. This research aims to contribute to a scientific approach to geo-collaboration in which the engineering of novel spatial decision support methods is complemented by a critical assessment of their utility in controlled, realistic experiments.
\end{abstract}

(c) 2008 Elsevier Ltd. All rights reserved.

\section{Introduction}

Argumentation mapping is a concept that allows for a discussion to be explicitly geographically referenced (Rinner, 1999, 2001, 2006). Argumentation Maps have the potential to supplement the planning process in order to facilitate efficient, meaningful, distributed, and asynchronous discussions. Keßler et al. (2005) introduced a software prototype as a proof of concept for argumentation mapping by integrating a Web-based GIS with an online discussion forum. We used this prototype in a quasi-naturalistic case study through which we analyze the utility of the Argumentation Map concept.

Utility is not a widely considered concept within GIS application development. It is often assumed that once a piece of software is distributed it will be utilized to its fullest. The Argumentation Map prototype produces a significant case to evaluate utility. The prototype was developed for the planning process, and therefore was designed to suit users with a wide variety of knowledge and skill levels. By evaluating the prototype this paper provides a better understanding of the utility of a specific geo-collaboration concept, thus contributing to participatory GIS research.

\footnotetext{
* Corresponding author. Tel.: +1 416979 5000x2686; fax +1 4169795362. E-mail address: rinner@geography.ryerson.ca (C. Rinner).
}

We start by reviewing the current state of GIScience research on the evaluation of geo-collaboration methods and tools with particular attention paid to the role of utility assessment (Section 2). Then, we identify the methodology for the Argumentation Map case study in terms of the type of case study, the software and data used, and the chosen approach to utility assessment (Section 3). In Section 4 , we present and discuss the results of the case study. The paper concludes with a summary and an outlook to future work (Section 5).

\section{Utility assessment in GIScience}

The utility of applications has drawn little attention in the body of literature in GIScience. We begin with a general definition of utility. The review continues by comparing a number of approaches for analyzing and criteria for describing the utility of GIS applications. Previous case studies where the utility of Public Participation GIS (PPGIS) has been assessed are also summarized.

The term utility originates and has been widely used within economics. It "has been historically used by economists to refer to personal feelings, such as pleasure, satisfaction, lack of pain" (CotoMillan, 2003: 7), which are generated in the consumer by a commercial product. This concept subsequently spun off profit maximization theory, which states that in order for a consumer to purchase a product, the cost must be less or equal to the utility it 
provides. Economists have applied this theory in a variety of ways, particularly in production and land value assessment. Utility in this paper describes the degree of success of an application in supporting a particular task. More specifically, we assess how well the Argumentation Map prototype facilitates an online, geographically referenced discussion.

Masser and Onsrud (1993) present a range of frameworks for understanding and analyzing successful implementation within PPGIS. Success can be viewed from two perspectives, that of the user and that of the application. Success for a user is described by the usability of the application, while success for the application is described by the application's utility (Masser and Onsrud, 1993). In addition to having two perspectives, the concept of success is context-specific, as "the difficulty in establishing the meaning of implementation success is inherent in the concept of multiple constituencies. Because these constituencies represent different roles and different needs, we would expect differences in the interpretation of success at any time" (Goodman, 1993: 49).

Ramasubramanian (1999) also considers success to be relative to the objectives and orientations of the organizations involved in a PPGIS implementation, while further developing the context that would allow for a successful implementation. Crucial characteristics for a successful PPGIS implementation include issue clarity, development of local knowledge, strategic actor relationships, and incremental problem resolution. While Ramasubramanian does not provide a framework for evaluating the successfulness of an application, she does introduce the social issues that can affect the implementation of a geo-collaboration tool.

Goodman (1993) describes the diffusion process of GIS within an organizational context. In doing so, two critical factors in the implementation process that promote successful adoption of the technology are identified: the paradox of value and the reward system. The paradox of value refers the concept that the achieved value of the implementation of a technology is often less than expected. Due to this discrepancy, Goodman encourages the designer not to overestimate the technology's benefits in order to foster accurate and firm commitment from the organization throughout the implementation process. The reward system is described as the degree to which benefits perceived in the organization are congruent to those the technology is set out to provide. Therefore, Goodman suggests that a more successful implementation process will be the result of the actual benefits of the technology more closely resembling the intended.

Laituri (2003) presents a method to assess PPGIS projects. She suggests that applications should be analyzed based on four components: context, connectivity, capabilities, and content. These components are then further broken down to provide units of investigation. While this paper presents a framework that helps to identify the objectives of an application, it does not examine how well the application achieves its objective.

Similarly to Laituri (2003), Jankowski and Nyerges (2001, 2003) present a formal structure for the scientific analysis of PPGIS applications. These authors refer to the analytic-deliberative decisionmaking process of Renn et al. (1995) that includes discursive and calculation elements. Jankowski and Nyerges present the Enhanced Adaptive Structuration Theory (EAST, version 2) which covers the convening, process, and output constructs of participatory decision processes. While their framework does not provide for the evaluation of the utility in PPGIS, it does support insight into the variables that influence how well an application works.

Peng and Tsou (2003) take a different approach to examining the success of an application, that of the quality of services. This approach introduces a number of concepts for investigation within Internet GIS, "including performance, scalability, functionality, portability, and security" (Peng and Tsou, 2003: 501). The disadvantage of this method is that it does not separate usability (user success) from utility (application success) for the implementation under investigation. As a result, the quality of services approach provides very little by way of evaluating how well the product is used. In addition to quality of services, Peng and Tsou (2003) examine the utility, versatility, and practicality of Internet GIS applications by presenting 12 case studies. These illustrate the opportunities and versatility of Internet GIS applications, but they do not explore how well the applications were employed by their users.

Nedovic-Budić (1997) describes four applications of GIS in local government agencies. The success of implementation and outcomes of implementation are related to the structure of the organization, resources available for implementation, and motivation for implementation. While Nedovic-Budić uses these cases to discuss implementation procedures, connections can surely be seen to utility studies, particularly in that the context or environment in which the implementation occurred has an effect on the outcome of the implementation.

Leitner et al. (2002) describe six types of GIS implementations for geo-collaboration: community-based GIS, university-community partnerships, GIS facilities in universities and public libraries, "map rooms", Internet map servers, and neighborhood GIS centers. The applications' appropriateness was evaluated on the basis of weighing the advantages versus the disadvantages. The Argumentation Map prototype shares most characteristics with the Leitner et al. Internet map server, particularly as it provides a service for distributed users. The Internet map server is described to have benefits as it allowed for direct contact with the spatial data, while also having drawbacks specifically that it is dependent on people and hardware available to the Internet map server provider, and users with enough knowledge to interpret the maps and data.

Kingston (2002) also analyzes Web-based PPGIS by considering the implementation of "Virtual Slaithwaite". The online availability and instantaneous updating were seen as some of the benefits. Kingston (2002) also noted a number of issues in the implementation, specifically training, Internet access, and copyright issues surrounding the data maintained in the system. From the Virtual Slaithwaite application it is concluded that online PPGIS provides a variety of benefits to the public participation process, but the author cautions that efforts must be made by the implementing body to increase access and training for the application to be successful overall.

The concept of utility assessment has been introduced in economics as consumer-based utility of products or services. Within GIScience, utility can be interpreted as the relation of costs to benefits associated with the use of a GIS. Therefore, a successful implementation of a GIS is one with a high utility. Further, the concept of success was discussed and it was determined that success, in GIScience is context-specific. That is to say each application will have its own characteristics that add to its success. The next section will introduce the case study and in doing so will illustrate what can be concluded to be a successful argumentation map, and therefore how utility can be determined for such applications.

\section{The Argumentation Map case study}

\subsection{Software prototype and case study}

The Argumentation Map prototype used in this case study combines a discussion forum with a basic mapping component (Keßler, 2004; Keßler et al., 2005). This Web-based application uses the GeoTools Lite mapping toolkit, a custom-built Java applet for the discussion forum, the MySQL database for storage of participant contributions, and the University of Minnesota MapServer for the supply of background map layers. Fig. 1 shows the use interface of the prototype during the case study. 


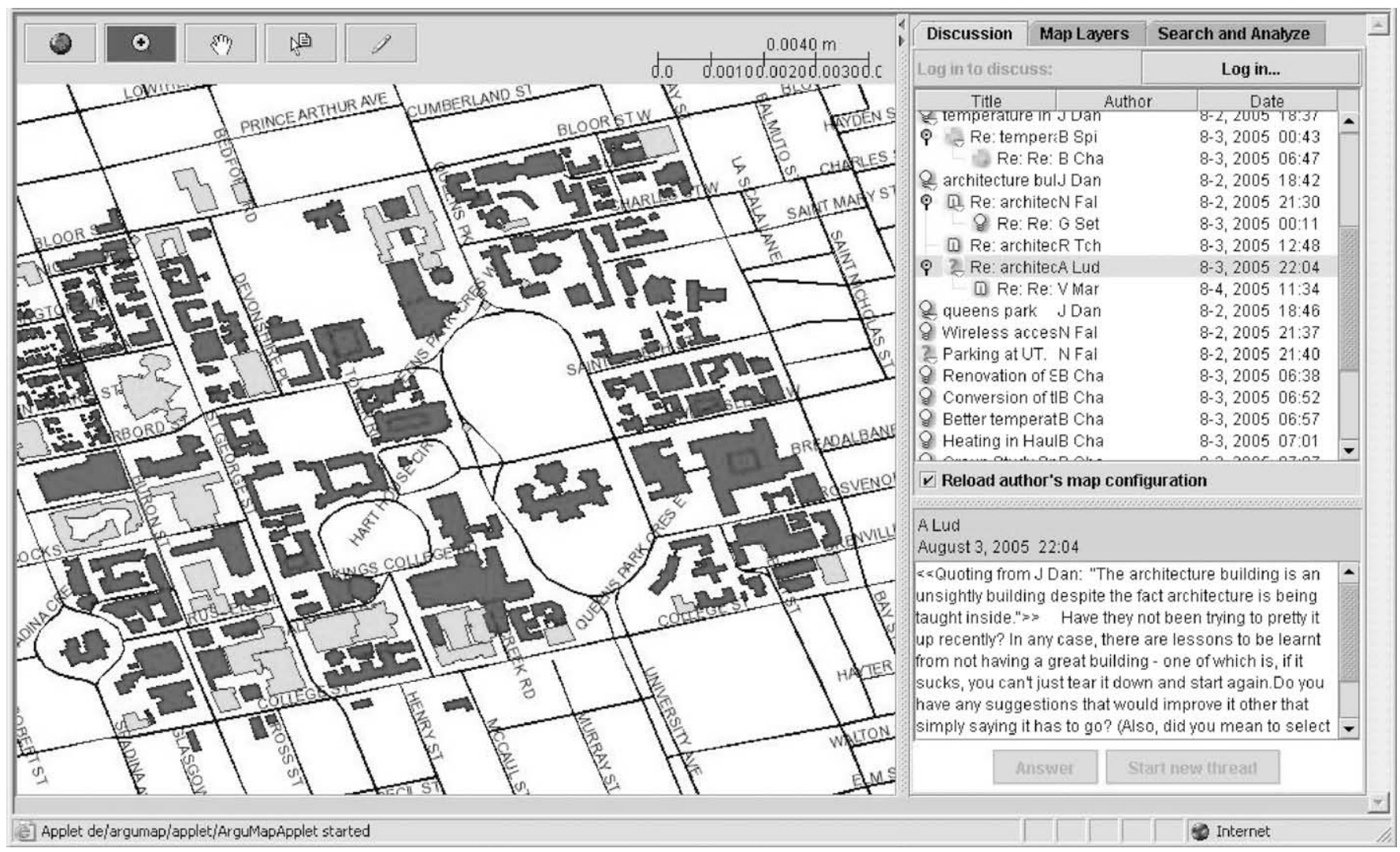

Fig. 1. The user interface of the Argumentation Map prototype during an ongoing discussion with geographic references highlighted on the map.

The prototype implements the Argumentation Map concept developed by Rinner (1999, 2001, 2006). Its functionality includes simple map navigation (zoom in/out, pan, zoom to full extent), layer management (switching of layers on and off), and the display of map labels (e.g. street names). In the discussion forum, contributions are represented by subject-author-date in a list and indented by discussion threads. A selected contribution is fully displayed in a text window. When selecting a contribution in the discussion forum, its geographic references will be highlighted on the map. In turn, when selecting a map element, all discussion contributions referring to this object will be highlighted in the list of contributions. Finally, the tool requires a login to enable users to respond to existing contributions or start a new discussion thread. While creating a contribution, geographic references can be selected in the map and will be stored together with the text of the message.

The relational MySQL database for the Argumentation Map prototype consists of seven tables (see Fig. 2). Two tables solely serve the purpose of describing the contribution type; contributiontypes table and geotypes table. Three other tables record data that is provided by the user; contributions table, coords table and members table. Finally, the last two tables relate the data tables to one another. These tables are the references table and the locations table.

The case study was formulated in order to assess both the usability and utility of the Argumentation Map prototype. The usability of the application refers to the users' opinion in regards to the functionality of the application, while the utility objectively reflects how the application was used. This paper summarizes the utility analysis, while the conclusions from the usability assessment were reported in Sidlar and Rinner (2007).

In preparation of the case study, various controls were applied in order to gain more information about the study participants, therefore resulting in a quasi-naturalistic study design. In particular, two questionnaires were distributed and required to be filled out by all participants, one pre-discussion and one post-discussion. Thirty-nine individuals were invited, of whom 11 agreed to participate in a one-week study to discuss ideas/concerns that they had with regards to the University of Toronto's St. George campus. From a statistical perspective this response rate cannot be considered to be representative, but in studies of this nature, it can be seen as a large sample. For example, Harrison and Haklay (2002) used an even smaller sample and argue that it serves as a 'typical' representation of the population as opposed to being representative (Harrison and Haklay, 2002: 845). Those who participated in the study were either given a group workshop or an individual introduction to the tool and the case study. In both cases the participants were given the same presentation that covered the basic functions of how to access the Argumentation Map, and how to login and make a contribution. The participants were then allowed to participate in the discussion freely, with no restrictions made on how much or when participation was to take place. The usability of the prototype is discussed in Sidlar and Rinner (2007) based on the participants' responses to the questionnaires. In contrast, this paper focuses on the utility analysis based on the participants' actual use of the tool.

\subsection{Method for utility assessment}

The discourse presented above illustrates that there is no clear methodology or set of criteria for evaluating the utility of PPGIS applications. The methodology used here builds upon two aspects of the literature: the concept of application success, and the need for adapted evaluation criteria. Key for this assessment is the trend that the definition of success changes in different applications, thereby complicating the creation of a general procedure for the analysis of utility. This trend therefore causes differences between 


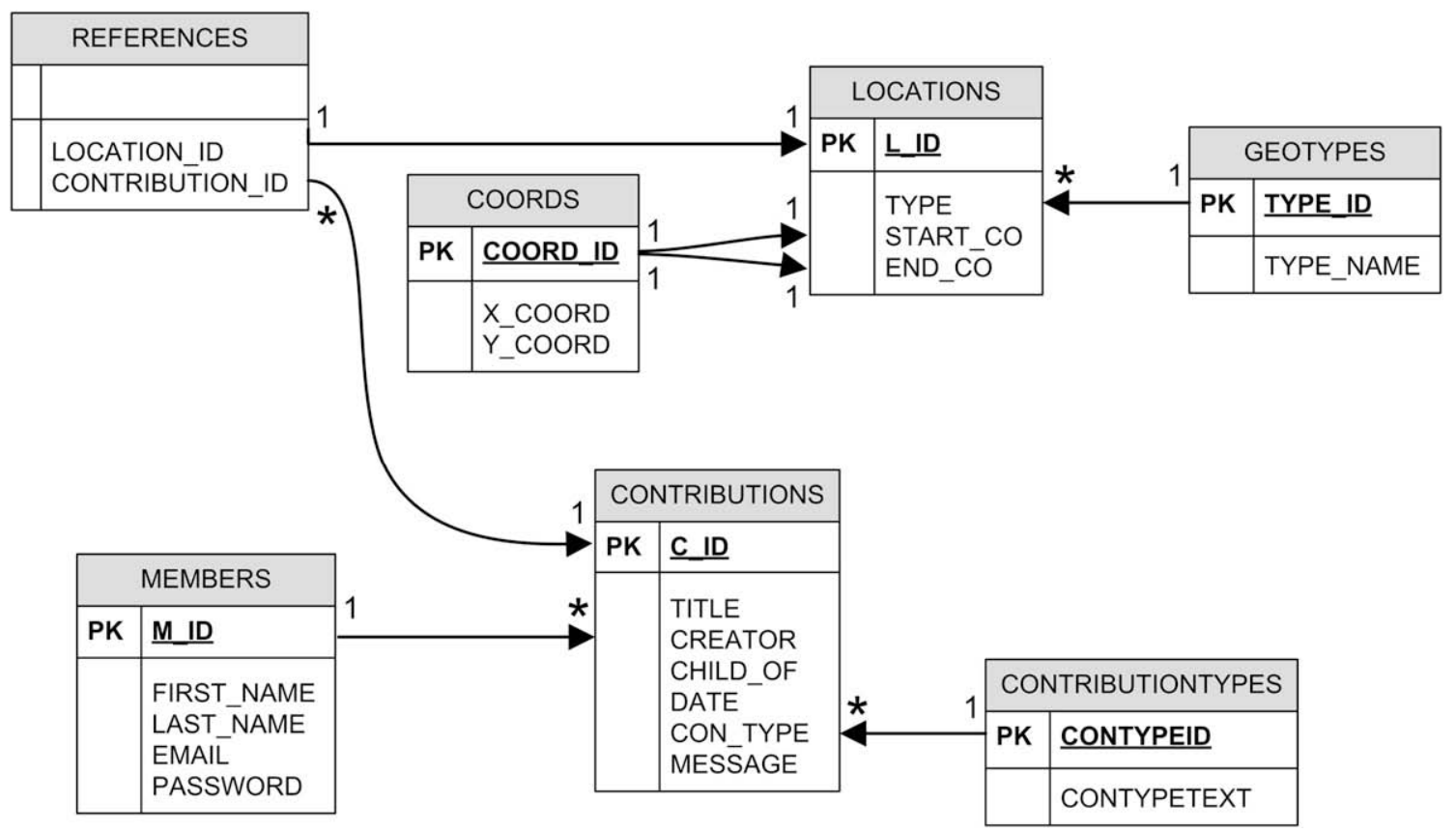

Fig. 2. The database schema for the Argumentation Map prototype (adapted from Keßler, 2004).

the methodologies used for evaluating success in different research projects.

This case study utilizes the concept of Goodman (1993) of paradox of value and reward system by producing a ratio that compares the actual benefits to the intended. From Nedovic-Budic (1997), Leitner et al. (2002), and Kingston (2002) the importance of identifying key characteristics of the application can be noted. This analysis therefore proposes the evaluation of utility based upon the distinct functions provided by the Argumentation Map application, i.e. accessing a map-based discussion forum, submitting discussion contributions at different logical levels in the forum, and providing geographic references together with contributions. Such an evaluation produces simple comparable ratios that easily identify success or failure on a component by component basis. Through the use of ratios the utility of the application is compared internally, therefore negating the social influences on implementations that are discussed by Ramasubramanian (1999).

This utility study investigates how well the functionality of the Argumentation Map prototype was employed. In order to do this, the first step is the extraction of geographically referenced discussion contributions. The data can then be analyzed by how they were created, what information they contain, and how they are related to each other. Since one of the major functionalities of the prototype is the connection of contributions to geographic references, this relationship will be analyzed in the utility study. The analysis also demonstrates the ideal use of the prototype.

The utility analysis focuses on evaluating the contributions made by the participants on the basis of three quantifiable criteria:

1. How many (geo)-graphic references were explicitly specified per discussion contribution compared to the potential references within the contributions?

2. Did respondents to a contribution re-use the references of the original contribution or did they provide additional references? How did the use of references differ throughout the replies?

3. Were references to existing map features more frequently used than references to user-specified point locations?
Generally, empirical values of success are difficult to set for these criteria, as Ramasubramanian (1999) and Goodman (1993) have shown that the success of a GIS implementation is contextspecific. In this analysis, success will be measured through the calculation of ratios of the actual use of argumentation mapping functions over the potential use of those functions. Therefore, the degree of success is measured by how close those ratios are to 1.0 .

\section{Summary of results}

\subsection{Participation statistics}

Table 1 presents a summary of the statistics related to the participation of the users during the case study. As previously stated, 11 individuals participated. During the one-week study, the participants wrote 60 contributions, which were organized in 20 discussion threads. In the 22 contributions with geographic references there were a total of 52 references made.

The timeline of the contributions and creation of new threads is illustrated in Fig. 3. Of the 60 contributions 22 included geographic references.

In an attempt to appreciate how the prototype was used, client IP addresses were retrieved from the Web server log file. The Web $\log$ reflects the participants' access of the study homepage and the Argumentation Map application. Here it was found that during the case study the prototype was accessed by 23 unique IP addresses.

Table 1

Summary statistics of the participation in the case study

\begin{tabular}{lr}
\hline Number of participants & 11 \\
Number of contributions & 60 \\
Number of threads & 20 \\
Number of contributions with references & 22 \\
Number of geographic references & 52 \\
Number of times prototype was accessed & 80 \\
Prototype access by unique IP addresses & 23 \\
\hline
\end{tabular}




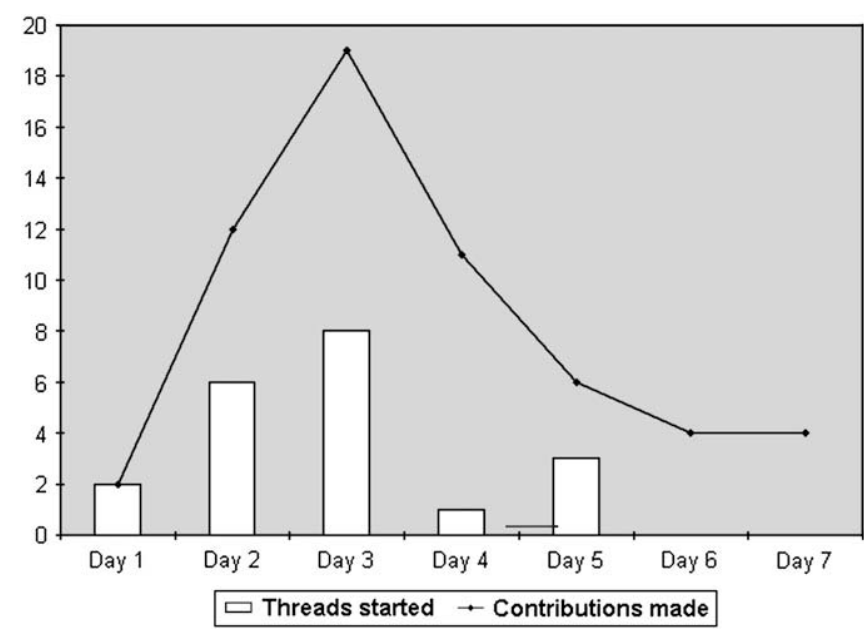

Fig. 3. Number of contributions including new threads per day during case study.

Further, by isolating the start-up page in the server log it could be determined that the prototype was loaded on 80 occasions. However, due to the client-server architecture of the prototype, it is not possible to gain information from the Web log about specific activities and the individual who performed a transaction. Therefore, the applicability of Web log analysis is somewhat constrained. The timeline of the contributions and the frequency of accessing the prototype are compared in Fig. 4.

As an exercise in data exploration, the total number of references was compared to the number of contributions with references in order to formulate a general interpretation into the extent in which the functions were used when they were used. This indicates that globally there were 2.4 references made per contribution. By considering the distribution of the references (Fig. 5) it becomes apparent that there exists one outlier-a contribution with 14 references. While it is important to show that contributions with this number of references are possible, such a contribution skews the overall figure. If this one contribution is removed the ratio significantly decreases to 1.8 references per contribution.

\subsection{Utility ratios}

The first ratio evaluates the combined use of the map and discussion forum, a key idea of the Argumentation Map concept. Before a ratio of utility can be calculated, the 38 contributions without references need to be examined to rule out any contributions where references would be nonsensical to be made. Among these contributions nine were written in a way that it was impossible for geographic references to be made. For example one participant asked whether "anybody got anything they wanna say about the parking situation at UT?" This contribution references the entire university campus, a reference that would not add anything to the discussion if it was made explicit. The utility calculation is then as follows: utility ratio $=$ [actual use]/[potential use] $=22$ / $(60-9)=22 / 51=0.43$.

The second criterion evaluates the replies in the discussion forum. These are of particular interest since they provide another aspect by which to investigate the depth of understanding and utilization of the Argumentation Map prototype. It should be noted that when a participant was replying to a contribution, the references made in the first contribution were not automatically copied, thus allowing the participant to fully shape the nature of their contribution. The first aspect of analyzing the use of references in the replies is to create a benchmark utility ratio for the

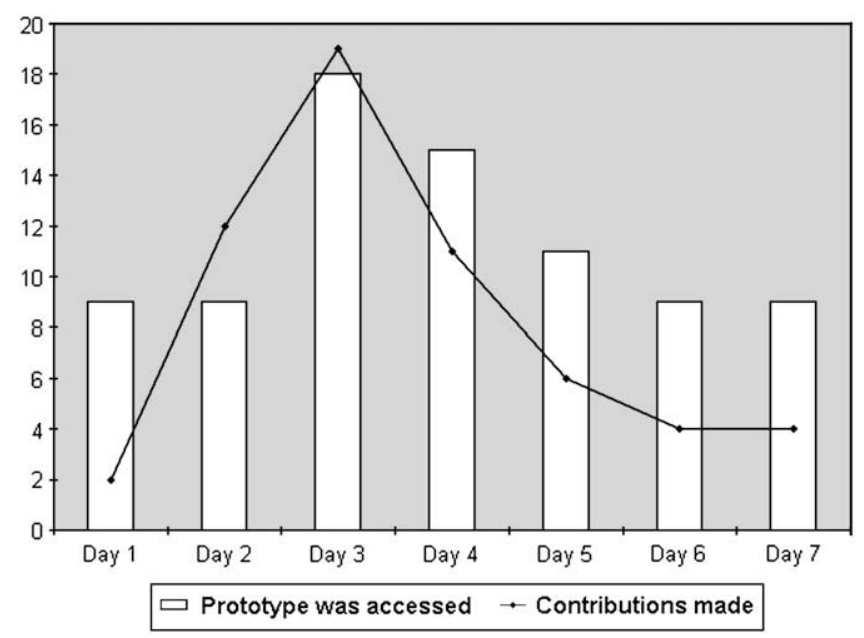

Fig. 4. Temporal distribution of contributions with the number of times the application was accessed.

contributions that began the threads (see Table 2). Similarly to the first utility calculation, the comments that could not include references were excluded from those with no references ( 1 out of 4 ) to calculate the total number of contributions. Therefore, dividing the number of contributions with references by the potential number of contributions with references results in a use ratio of 0.84 .

Now that we understand the use of references in the initial contributions, the use in the replies can be put into context. We begin by examining the first degree of replies (replies to the contributions that began the discussion threads), as presented in Table 2. After removing the three contributions that could not have sensible references there were a total of 20 first-degree replies. The use ratio of the number of references in the first-degree replies is 0.15. This illustrates a steep decline in the use of the map functions, specifically references, after the first level of contribution. It can be noted that no references that were made in the initial contribution were repeated in the replies. This method can be likewise completed for second- and third-degree replies (Table 2) producing use ratios of 0.25 and 0.5 , respectively.

The increases in these ratios can be partially explained by the small amount of second- and third-degree replies, and therefore the ratios may be skewed by small absolute changes. Overall, through a use ratio comparison it can be seen that there is a clear discrepancy in the use of references in the initial contributions and their subsequent replies. Therefore it can be concluded that the participants were generally relying on the reference(s) associated with the initial contribution when they made their replies. Further, the repetition of references in the second- and third-degree replies is uncommon, as there is only one reply with a repeated reference and that is a second-degree reply. The result is a distribution that decreases as the degree of reply increases.

The third utility criterion considers how engaged the participants were to include references in their contributions. The level of engagement is investigated by the types of references which were made by the users. This version of the prototype allowed the users to add references in two ways: by selecting a map feature, or by drawing a point. The data for this criterion displayed a clear preference as there were five user-specified point annotations on the map, while there were 47 selections of existing map features as geographic references. The 47 selected features could have been two possible feature types, buildings or road segments. However, there were only three road segments selected while the rest of the references were made to buildings. 


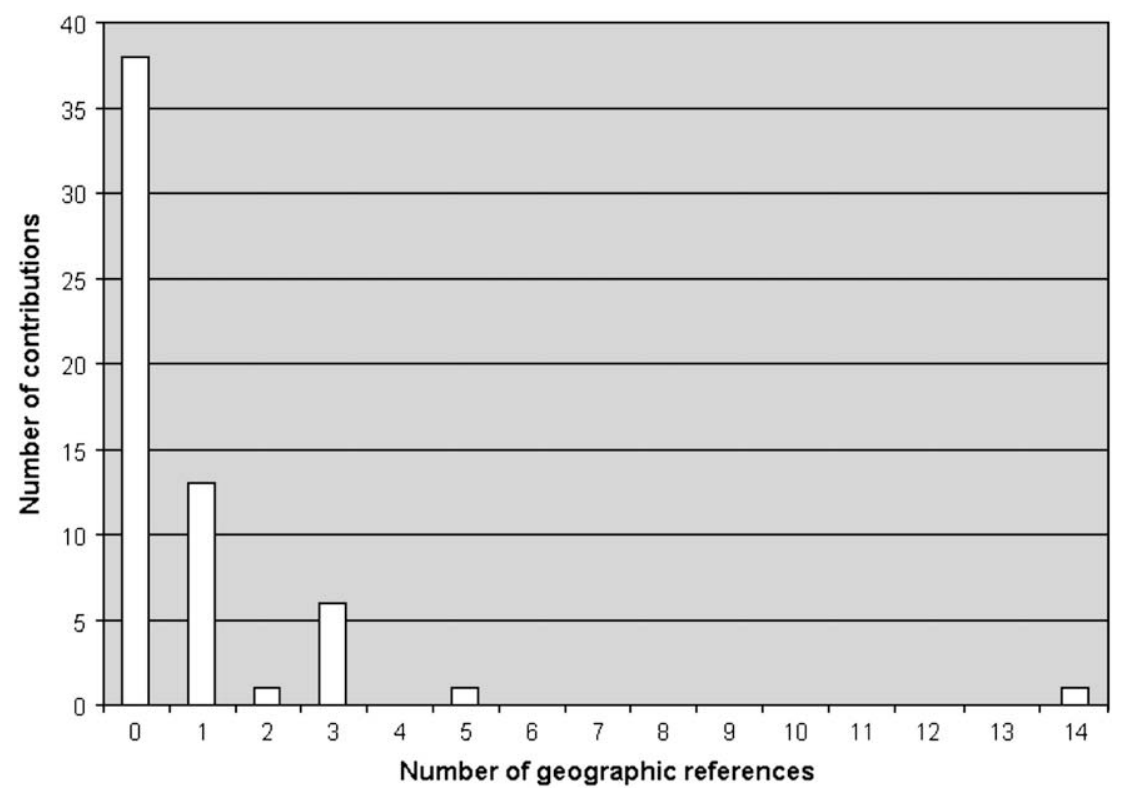

Fig. 5. Number of contributions by number of geographic references.

\section{Discussion}

The timeline of contributions being registered (Fig. 3) describes the apprehension of the participants to begin the discussion. On the first day only two contributions were made, although the prototype was accessed nine times (Fig. 4). On day three, the rate of making contributions peaks. Unlike the first day the number of contributions exceeds the number of times the prototype was accessed, indicating that by this point in the discussion process participants were making more than one contribution per visit. After this point the number of new threads declines substantially. This decline is followed by a decline and stabilization in the number of contributions and the number of times the prototype was accessed. This decline and stabilization seem to reflect a decline in enthusiasm to continue with the week-long study, which can largely be attributed to feelings of lack of empowerment due to the quasi-naturalistic approach in the study.

One objective of including a consideration of the Web server log file is to gain understanding into the behavior of the participants, in particular, from which location they were contributing to the discussion. The conclusions that can be drawn from such an analysis are somewhat mitigated by the prevalence of dynamic IP addresses.

With respect to the utility assessment of the Argumentation Map concept, the first criterion analyzed the use of the map with the discussion forum. The evaluation produced an overall utility ratio of 0.43 . Initially this indicates a large percentage of contributions that did not explicitly refer to places on the map.

The second criterion investigated this finding further by considering upon which level of replies in the discussion the map references were most prevalent. Eighty-four percent of the initial contributions had references, while the use of references significantly decreased throughout the reply levels. Although there were

Table 2

Cross-tabulation of contribution frequency by type and by number of references

\begin{tabular}{lrllllll}
\hline & \multicolumn{7}{l}{ Number of references } \\
\cline { 2 - 8 } & 0 & 1 & 2 & 3 & 4 & 5 & 14 \\
\hline New threads & 4 & 9 & 1 & 5 & 0 & 1 & 0 \\
1st degree reply & 20 & 1 & 0 & 1 & 0 & 0 & 1 \\
2nd degree reply & 9 & 2 & 0 & 0 & 0 & 0 & 0 \\
3rd degree reply & 3 & 1 & 0 & 0 & 0 & 0 & 0 \\
\hline
\end{tabular}

only a small number of replies, which may have skewed the subsequent ratios, we can conclude from the thread-level ratio of 0.84 that the ability to specify geographic references with discussion contributions was utilized. Further, the case study was limited to a one-week study period, and therefore the short period of interaction with the prototype may not have been long enough to allow for all participants to fully utilize the prototype.

The third criterion found that different types of geographic references were utilized although with a strong preference towards selecting existing map features, and in particular the building polygons. The participants' choices with respect to the type of reference and feature type were limited to the options implemented in the specific map of the case study. In order to examine whether the participants were content with the features provided, we asked in the post-discussion questionnaire whether they wanted a function that was not included in the prototype. The answers generally dealt with the discussion forum, while there was one user that wished to enhance the ability to provide a reference by allowing for the delimiting of "non-building features".

Overall, the use of the integrated map with the discussion forum could clearly be demonstrated although this use varied between different levels of discussion. Therefore, this case study indicates the utility of the Argumentation Map concept as implemented in the Keßler et al. (2005) prototype. It must be noted that the small sample size did not permit a rigorous statistical analysis of these results.

\section{Conclusions}

In the development of geo-collaboration tools, the usability of a given application is increasingly evaluated by researchers while its utility is often taken as a given. This study addresses this implicit assumption by developing a framework for investigating participatory GIS utility and applying the framework to the Argumentation Map prototype. The framework is based on two aspects that were developed from previous work: the definition of the success of an application, and an in-depth consideration of the specific functions offered by the particular application. This approach was then applied to the Argumentation Map prototype.

This case study defined the level of success by using ratios that could be both interpreted independently of one another, or in combination. The ratios were then calculated on three key aspects 
of the Argumentation Map that would reflect its utility. From these calculations it could be concluded that the prototype's functionality was utilized in all aspects, although not always to its fullest. We attribute a lack of engagement to the short time period of interaction allowed with the prototype. The paper also discusses the concerns that were raised by the participants with respect to the functions of the tool. Future case studies should therefore be conducted over a longer period of time, and attempt to incorporate a larger audience to provide statistical significance.

In summary, this paper has illustrated a quasi-naturalistic case study using an Argumentation Map prototype. A simple framework for the assessment of the utility of a participatory spatial decision support tool has been presented. This work contributes to a more comprehensive approach to evaluating the usefulness of geocollaboration tools by adding utility concerns to the predominant examination of software usability.

\section{Acknowledgments}

We would like to thank the participants in the case study for their contribution to this research. We are indebted to Carsten Keßler for his support in adapting and setting up the Argumap application for the case study and Lauren Beharry for her valuable help with preparing the manuscript. Partial funding from the Natural Sciences and Engineering Research Council of Canada (NSERC) and the GEOIDE Network of Centres of Excellence in Geomatics, project HSS-DSD\#17, "Promoting Sustainable Communities through Participatory Spatial Decision Support”, is gratefully acknowledged.

\section{References}

Coto-Millan, P., 2003. Utility and Production: Theory and Applications. Physica, Heidelberg, New York.

Goodman, P., 1993. Implementation of new information technology. In: Masser, I., Onsrud, H. (Eds.), Diffusion and Use of Geographic Information Technologies. Kluwer, Boston, pp. 45-58.

Harrison, C., Haklay, M., 2002. The Potential of Public Participation Geographic Information Systems in UK Environmental Planning: Appraisals by Active Publics. Journal of Environmental Planning and Management 45 (6), 841-863.
Jankowski, P., Nyerges, T., 2001. GIS-Supported Collaborative Decision Making: Results of an Experiment. Annals of the Association of American Geographers 91 (1), 48-70.

Jankowski, P., Nyerges, T., 2003. Toward a Framework for Research on Geographic Information-Supported Participatory Decision-Making. URISA Journal 15 (APA II), 9-17.

Keßler, C., 2004. Design and Implementation of Argumentation Maps. Diploma Thesis, Westfälische Wilhelms-Universität, Münster, Germany. Available online at http://www.carstenkessler.de/argumap/diploma.pdf [24 August 2007.].

Keßler, C., Rinner, C., Raubal, M., 2005. An Argumentation Map Prototype to Support Decision-Making in Spatial Planning. In: Toppen, F., Painho, M. (Eds.), Proceedings of AGILE 2005-8th Conference on Geographic Information Science, pp. 135-142. 26-28 May 2005, Estoril, Portugal.

Kingston, R., 2002. Web-based PPGIS in the United Kingdom. In: Craig, W., Harris, T., Weiner, D. (Eds.), Community Participation and Geographic Information Systems. Taylor and Francis, New York, pp. 101-112.

Laituri, M., 2003. The Issue of Access: An Assessment Guide for Evaluating Public Participation Geographic Information Science Case Studies. URISA Journal 15 (APA II), 25-32.

Leitner, H., McMaster, R., Elwood, S., McMaster, S., Sheppard, E., 2002. Models for making GIS available to community organizations: dimensions of difference and appropriateness. In: Craig, W., Harris, T., Weiner, D. (Eds.), Community Participation and Geographic Information Systems. Taylor and Francis, New York, pp. 37-52.

Masser, I., Onsrud, H., 1993. Diffusion and Use of Geographic Information Technologies. Kluwer, Boston.

Nedovic-Budić, Z., 1997. GIS Technology and Organisational Context: Interaction and Adaptation. In: Craglia, M., Couclelis, H. (Eds.), Geographic Information Research: Bridging the Atlantic. Taylor and Francis, Bristol, PA, pp. 165-184.

Peng, Z.-R., Tsou, M.-H., 2003. Internet GIS. John Wiley, New York.

Ramasubramanian, L., 1999. GIS implementation in developing countries: learning from organizational theory and reflective practice. Transactions in GIS 3 (4), 359-380.

Renn, O., Webler, T., Wiedemann, P., 1995. Fairness and Competence in Citizen Participation: Evaluating Models for Environmental Discourse. Kluwer, Dordrecht.

Rinner, C., 1999. Argumentation Maps-GIS-based Discussion Support for Online Planning. GMD Research Series No. 22. GMD German National Research Center for Information Technology, Sankt Augustin, Germany. Available online at http://publica.fraunhofer.de/eprints/B-73065.pdf (24 August 2007.).

Rinner, C., 2001. Argumentation maps: GIS-based discussion support for on-line planning. Environment and Planning B: Planning and Design 28 (6), $847-863$.

Rinner, C., 2006. Argumentation mapping in collaborative spatial decision making In: Balram, S., Dragicevic, S. (Eds.), Collaborative Geographic Information Systems. Idea Group, Hershey, pp. 85-102.

Sidlar, C., Rinner, C., 2007. Analyzing the usability of an argumentation map as a participatory spatial decision support tool. URISA Journal 19 (1), 47-55. 